OPEN ACCESS

Edited by:

Gustavo Henrique Goldman,

University of São Paulo, Brazi

Reviewed by:

Ming-Guang Feng,

Zhejiang University, China

Yun Chen,

Zhejiang University, China

${ }^{*}$ Correspondence:

Guanghui Wang

wgh2891458@163.com

Specialty section:

This article was submitted to Fungi and Their Interactions,

a section of the journal

Frontiers in Microbiology

Received: 11 February 2019

Accepted: 29 April 2019

Published: 22 May 2019

Citation:

Ren J, Li C, Gao C, Xu J-R Jiang $C$ and Wang $G$ (2019) Deletion

of FgHOG1 is Suppressive to the mgv1 Mutant by Stimulating Gpmk1 Activation and Avoiding Intracellular

Turgor Elevation in Fusarium

graminearum

Front. Microbiol. 10:1073

doi: 10.3389/fmicb.2019.01073

\section{Deletion of FgHOG1 Is Suppressive to the mgv1 Mutant by Stimulating Gpmk1 Activation and Avoiding Intracellular Turgor Elevation in Fusarium graminearum}

\author{
Jingyi Ren ${ }^{1}$, Chengliang $\mathrm{Li}^{1}$, Chengyu Gao ${ }^{1}$, Jin-Rong $X u^{2}$, Cong Jiang ${ }^{1,2}$ and \\ Guanghui Wang ${ }^{1,2 *}$ \\ 1 Purdue-NWAFU Joint Research Center, College of Plant Protection, Northwest A\&F University, Yangling, China, \\ ${ }^{2}$ Department of Botany and Plant Pathology, Purdue University, West Lafayette, IN, United States
}

Fusarium head blight caused by Fusarium graminearum is an important disease of wheat and barley. Previous studies have showed that all three MAP kinase genes, MGV1, FgHOG1, and GPMK1, are involved in regulating hyphal growth, sexual reproduction, plant infection, and stress responses in this pathogen. To determine the relationship between the Mgv1 and FgHog1 pathways, in this study, we generated and characterized the mgv1 Fghog1 double mutant. Deletion of FgHOG1 partially rescued the defects of the mgv1 mutant in vegetative growth and cell wall integrity but had no effects on its defects in plant infection and DON production. The mgv1 Fghog1 mutant grew faster and was more tolerant to cell wall stressors than the mgv1 mutant. Swollen compartments and cell burst were observed frequently in the mgv1 mutant but rarely in the mgv1 Fghog1 mutant when treated with fungicide fludioxonil or cell wall stressor Congo red. Conversely, the deletion of MGV1 also alleviated the hyperosmotic sensitivity of the Fghog1 mutant in vegetative growth. TGY assays indicated increased phosphorylation of FgHog1 in the mgv1 mutant, and TEY assays further revealed elevated activation of Gpmk1 in the mgv1 Fghog1 double mutant, particularly under cell wall stress conditions. Overall, our data showed that deletion of FgHOG1 partially suppressed the defects of the mgv1 mutant, possibly by affecting genes related to cell wall integrity and osmoregulation via the over-activation of Gpmk1 MAP kinase and avoiding intracellular turgor elevation.

Keywords: cell wall integrity, osmoregulation, Fusarium graminearum, Gpmk1 MAP kinase, intracellular turgor

\section{INTRODUCTION}

Fusarium head blight, a devastating disease of wheat and barley, is caused by the filamentous ascomycete Fusarium graminearum. In addition to severe yield losses, infested grains are often contaminated with mycotoxins, including deoxynivalenol (DON) and zearalenone (Jiang et al., 2016; Boenisch et al., 2017). F. graminearum also causes stalk and ear rots on maize and infects other small grains (Bai and Shaner, 2004; Goswami and Kistler, 2004). It overwinters and produces 
perithecia on plant debris. Ascospores discharged from perithecia are the primary inoculum to infect flowering wheat heads (Trail, 2009). F. graminearum also produces asexual spores known as conidia that are infectious and important for pathogen spreading.

Like other filamentous ascomycetes, F. graminearum has three well-conserved mitogen-activated protein (MAP) kinase cascades that are involved in regulating various developmental and infection processes in fungal pathogens (Wang et al., 2011). $M G V 1$, the first MAP kinase (MAPK) gene characterized in F. graminearum, is orthologous to the cell wall integrity (CWI) MAPK SLT2 in the budding yeast and MPS1 in Magnaporthe oryzae (Xu et al., 1998; Hou et al., 2002). Deletion of MGV1 causes severe defects in vegetative growth and plant infection. The $m g v 1$ mutant is almost non-pathogenic and is significantly reduced in hyphal growth and DON production. $M G V 1$ is important for CWI, and deletion of $M G V 1$ results in hypersensitivity to cell wall stress or lytic enzymes. The mgv1 mutant is blocked in hyphal fusion, and it is female sterile during sexual reproduction (Hou et al., 2002).

The other two MAPK genes, GPMK1 (FMK1) and FgHOG1, also have been functionally characterized in F. graminearum (Jenczmionka et al., 2003; Zheng et al., 2012). GPMK1, an ortholog of yeast FUS3/KSS1 and M. oryzae PMK1 (Xu and Hamer, 1996), is essential for regulating sexual and asexual production, plant infection processes, and the expression of genes encoding secreted lytic enzymes (Jenczmionka et al., 2003; Urban et al., 2003). The gpmk1 deletion mutant is reduced in growth rate but blocked in perithecium formation. Whereas Mgv1 and Gpmk1 are two MAPKs with the TEY dual-phosphorylation site, FgHog1, the third MAPK in F. graminearum has the TGY motif. FgHog1 is orthologous to yeast Hogl of the high-osmolarity glycerol (HOG) pathway and M. oryzae Osml (Dixon et al., 1999; Saito and Tatebayashi, 2004). Deletion of FgHOG1 results in a minor reduction in vegetative growth but significantly reduced DON production. The Fghog1 mutant is defective in response to hyperosmotic and oxidative stresses but resistant to fludioxonil fungicides that cause the overstimulation of the HOG pathway and increase in intracellular turgor in fungi (Zheng et al., 2012; Segorbe et al., 2017).

Interestingly, all three MAPK pathways appear to be involved in regulating growth, plant infection, responses to different stresses, and sexual reproduction (Wang et al., 2011). However, their functional relationship and possible cross-talking among different pathways have not been well studied in F. graminearum. It was previously reported that the deletion of $M K K 1$ (the MAPKK of the CWI pathway) significantly reduced the phosphorylation level of FgHog1 (Yun et al., 2014), suggesting a cross talk between the HOG pathway and the CWI pathway. In this study, we generated and characterized the mgv1 Fghog1 double mutant. Deletion of FgHOG1 partially rescued the defects of the $m g v 1$ mutant in vegetative growth, CWI, and responses to elevated temperature but had no effect on its defects in plant infection and DON production. Interestingly, the $m g v 1$ mutant was hypersensitive to fludioxonil, which also could be partially suppressed by deletion of FgHOG1. Furthermore, the mgv1 Fghog1 mutant had an increased phosphorylation level of Gpmk1. Overall, our results showed that deletion of FgHOG1 partially suppressed some of the defects of the mgv1 mutant, possibly by increasing the activation of Gpmk1 and avoiding the upsurge of intracellular turgor.

\section{RESULTS}

\section{Deletion of FgHOG1 Partially Suppressed the Growth but Not the Virulence Defect of the mgvi Mutant}

To generate the mgv1 Fghog1 double mutant, the FgHOG1 gene replacement construct carrying the geneticin resistance marker (NEO) was generated and transformed into the mgv1 mutant. Transformants resistant to both hygromycin B and geneticin were isolated and screened by PCR for the deletion of FgHOG1 (Supplementary Figure S1). Two mgv1 Fghog1 mutant strains, MH17 and MH18, were identified, and they had the same phenotypes described below, although only data with strain MH18 were presented. To our surprise, the mgv1 Fghog1 mutant grew faster than the mgv1 mutant (Figure 1A). When assayed with race tube cultures grown on CM (complete medium), the $m g v 1$ mutant grew at approximately $2.7 \mathrm{~mm} /$ day, but the growth rate of the $m g v 1$ Fghog1 mutant was over $4.0 \mathrm{~mm} /$ day (Table 1), which was approximately a $48 \%$ increase in comparison with $m g v 1$. These results indicate that deletion of $\mathrm{FgHOG} 1$ partially suppressed the growth defect of the mgv1 mutant. However, in infection assays with wheat heads, the mgv1 Fghog1 mutant, like the $m g v 1$ and Fghog1 mutants, rarely caused limited necrosis on glumes but never caused typical symptoms on the inoculated

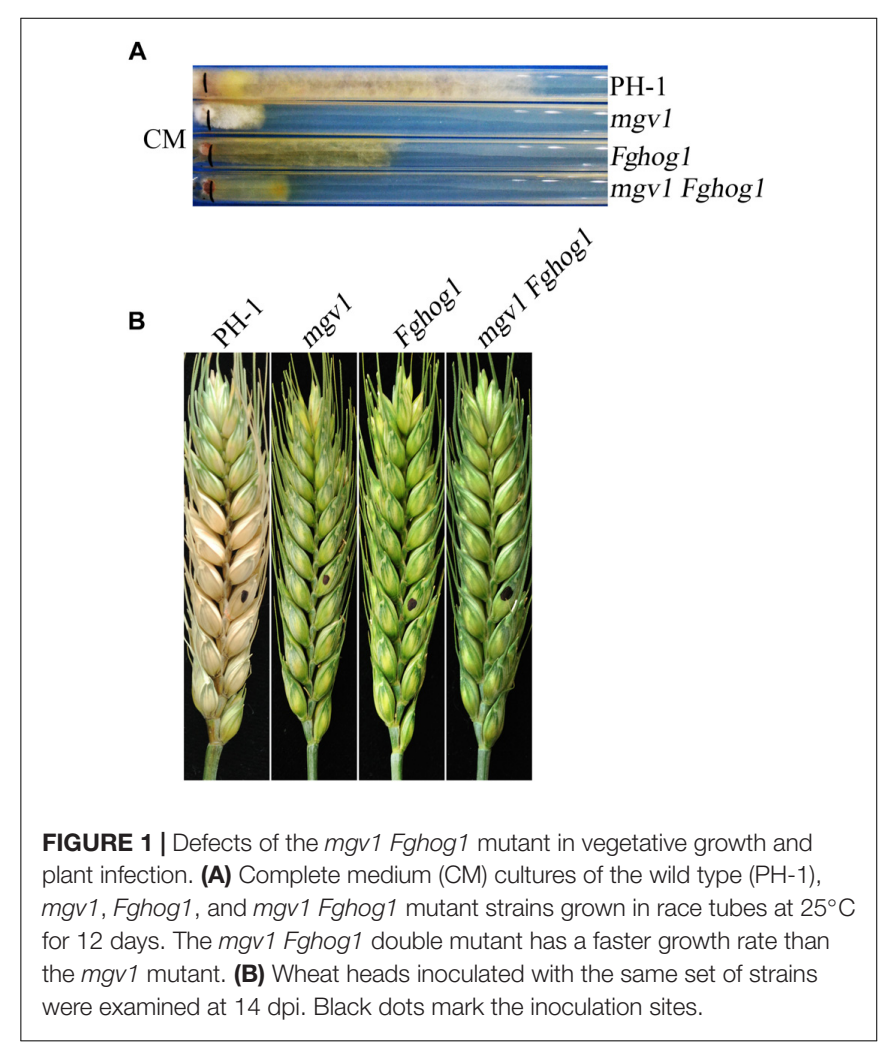


TABLE 1 | Phenotype characterization of the mutants used in this study.

\begin{tabular}{|c|c|c|c|c|}
\hline Strains & $\begin{array}{l}\text { Growth Rate a } \\
\text { (mm/day) }\end{array}$ & $\begin{array}{l}\text { Disease } \\
\text { index }^{b}\end{array}$ & $\begin{array}{l}\text { DON } \\
\text { production }^{c} \\
\text { (ppm) }\end{array}$ & $\begin{array}{l}\text { Release of } \\
\text { protoplasts }{ }^{d} 10^{5} \\
\text { protoplasts } / \mathrm{mL} \text { ) }\end{array}$ \\
\hline $\mathrm{PH}-1$ (WT) & $8.4 \pm 0.6^{A *}$ & $10.3 \pm 2.6^{A}$ & $124.3 \pm 21.5$ & $9.3 \pm 2.4^{\mathrm{A}}$ \\
\hline C6 (mgv1) & $2.7 \pm 0.0^{C}$ & $0^{\mathrm{B}}$ & nd & $61.8 \pm 3.3^{\mathrm{B}}$ \\
\hline HG15 (Fghog1) & $8.2 \pm 0.4^{A}$ & $0^{\mathrm{B}}$ & nd & $2.7 \pm 3.8^{C}$ \\
\hline MH18 (mgv1 Fghog1) & $4.0 \pm 0.2^{\mathrm{B}}$ & $0^{\mathrm{B}}$ & nd & $2.9 \pm 8.8^{C}$ \\
\hline
\end{tabular}

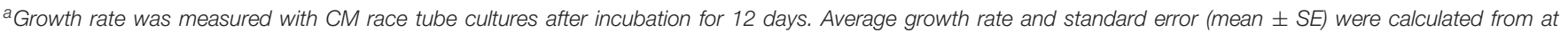

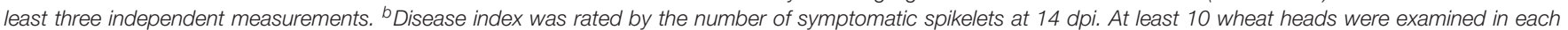

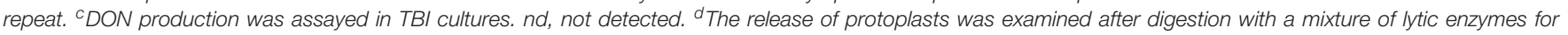

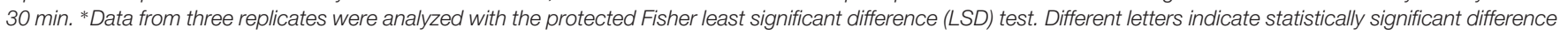
$(P=0.05)$.

kernels or spread infection to neighboring spikelets (Figure 1B and Table 1). Under the same conditions, the wild type strain $\mathrm{PH}-1$ caused severe head blight symptoms in inoculated wheat heads and had a disease index of over 10 (Figure 1B and Table 1). We also performed DON production assays in TBI cultures and found that the DON was not detectable in the mgv1 Fghog1 double mutant as well as in the $m g v 1$ and Fghog1 single mutants (Table 1). Therefore, deletion of FgHOG1 in the $m g v 1$ mutant had no suppressive effect on its virulence defect.

\section{The mgv1 Fghog1 Mutant Is More Tolerant to Cell Wall Stresses Than the mgv1 Mutant}

It has been reported that the $m g v 1$ mutant is hypersensitive to cell wall-degrading enzymes (Hou et al., 2002). To determine whether the inactivation of FgHog1 could recover other defects of the mgv1 mutant, germlings harvested from $12 \mathrm{~h}$ YEPD (yeast extract-peptone-dextrose medium) cultures were treated with a mixture of cell wall lytic enzymes for $30 \mathrm{~min}$. In the $m g v 1$ mutant, abundant protoplasts were produced, while germlings from the wild type strain PH-1 released few protoplasts (Figure $\mathbf{2 A}$ and Table 1), which is consistent with the previous study (Hou et al., 2002). However, under the same conditions, the Fghog1 and $m g v 1$ Fghog1 mutants released fewer protoplasts than $m g v 1$ (Figure $\mathbf{2 A}$ and Table $\mathbf{1}$ ). These results show that deletion of FgHOG1 also alleviated the hypersensitivity of the mgv1 mutant to cell wall lytic enzymes.

To confirm the partial recovery of $m g v 1$ Fghog1 in cell wall defects, we then assayed hyphal growth on CM with $300 \mu \mathrm{g} / \mathrm{ml}$ Congo red (CR), a compound interfering with the fungal cell wall (Merzendorfer, 2013). The inhibition rate on colony growth by CR was $41.9 \%$ in the mgv1 Fghog1 double mutant, which was significantly lower than that $(74.0 \%)$ of the $m g v 1$ mutant (Table 2 and Figure 2B). We also assayed the effects of CR on conidial germination. After incubation in regular YEPD for $6 \mathrm{~h}$, conidia of the double mutant had no obvious defects in germination in comparison with $\mathrm{PH}-1$ or the mgv1 or Fghog1 mutant (Figure 2C). However, in the presence of as low as $15 \mu \mathrm{g} / \mathrm{ml} \mathrm{CR}$, the conidial germination rate was only $14 \%$ in the $m g v 1$ mutant, although abnormal swelling was observed in some conidium compartments (Figure 2C and Table 2). Under the same conditions, the rate of conidial germination was not affected in the strains of $\mathrm{PH}-1$ and Fghog1 mutant, but reduced to $78.8 \%$ in the double mutant (Table 2). Germ tubes of the Fghog1 and mgv1 Fghog1 mutants also tended to be shorter than those of PH-1 (Figure 2C). These data further indicate that deletion of FgHOG1 suppressed the CWI defect of the mgv1 mutant. Abnormal swelling in the $m g v 1$ mutant may be caused by the stimulation of FgHog1 by CR treatment, leading to an increase in intracellular turgor.

\section{Cell Wall Deposition Is Affected in the mgv1 Fghog1 Mutant}

Calcofluor white (CFW) is a commonly used stain to visualize the fungal cell wall. When germlings harvested from $12 \mathrm{~h}$ YEPD cultures were stained with $50 \mu \mathrm{g} / \mathrm{ml} \mathrm{CFW}$, fluorescence signals were evenly distributed along the cell wall and septa in the wild type and mgv1 mutant (Figure 3A). However, we noticed that CFW staining often resulted in the burst of germ tubes at the tip of the mgv1 mutant during microscopic examination, suggesting a weakened cell wall at the tips. In the mgv1 Fghog1 mutant, bright spots of CFW staining were observed in many germlings but not in conidium compartments (Figure 3A). Weaker and fewer CFW staining spots also were observed in germlings of the Fghog1 mutant (Figure 3A). These data indicate that deletion of FgHOG1 caused uneven deposition of cell wall, especially in the mgv1 mutant.

We also assayed the effect of CFW on conidial germination. In the presence of $7.5 \mu \mathrm{g} / \mathrm{ml} \mathrm{CFW}$, the conidial germination rate of the mgv1 mutant was lower than $1 \%$, while that of the mgv1 Fghog1 double mutant was increased to $40 \%$ (Table 2). Interestingly, the $m g v 1$ mutant had empty conidium compartments besides swollen compartments (Figure 3B). Under the same conditions, empty compartments in conidia were rarely observed in the $m g v 1$ Fghog1 double mutant (Figure 3B). Empty compartments observed in the mgv1 mutant may be caused by cell burst resulting from a weakened cell wall and elevated intracellular turgor. However, when we used a higher concentration $(15 \mu \mathrm{g} / \mathrm{ml})$ of CFW to treat the conidia, we found that all conidia of both the mgv1 and mgv1 Fghog1 mutants were unable to germinate and had empty conidium compartments (Figure 3C). These data indicate that the mgv1 Fghog1 double mutant was still defective in CWI, although deletion of FgHOG1 partially rescued the cell wall defect of the $m g v 1$ mutant. 
A

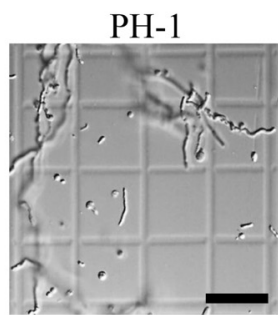

PH-1

B
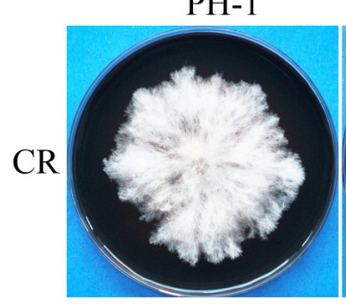

C

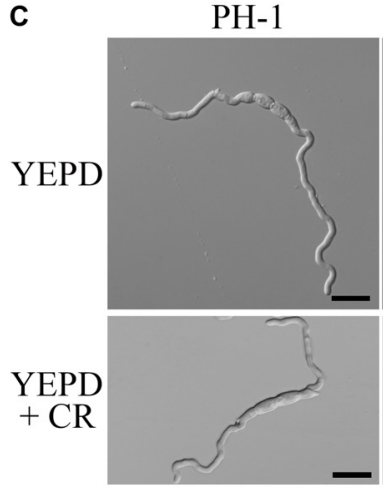

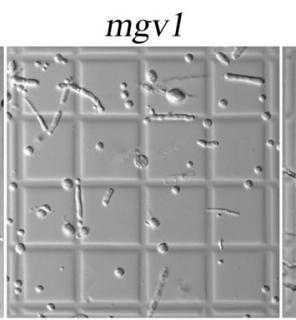
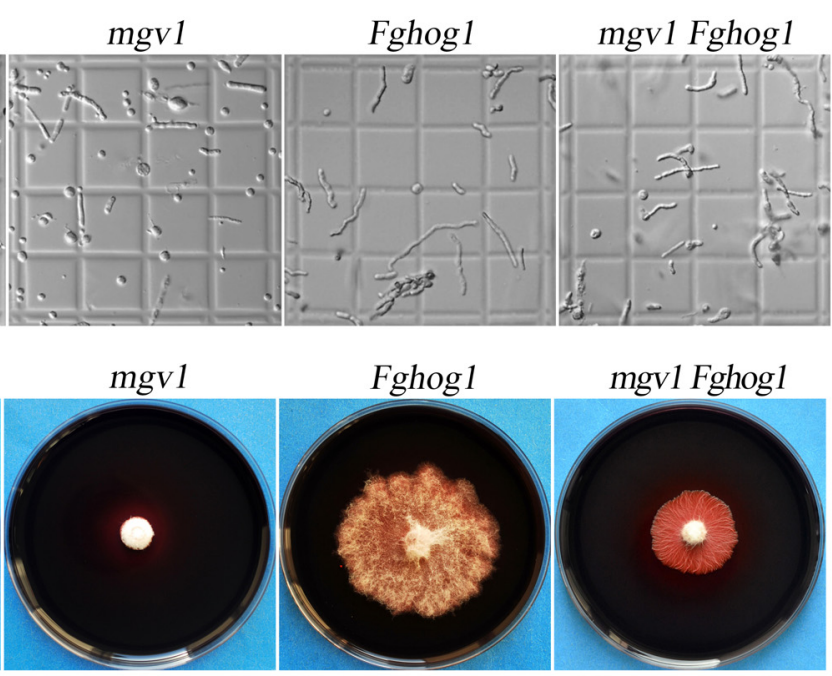

mgv1

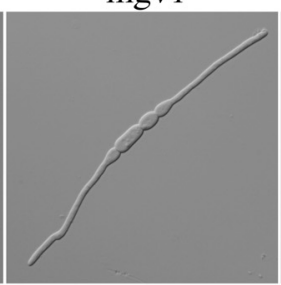

Fghog1

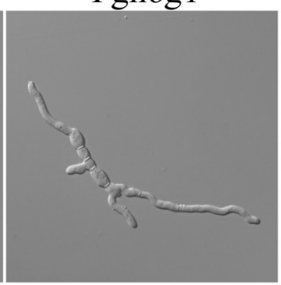

mgv1 Fghog1
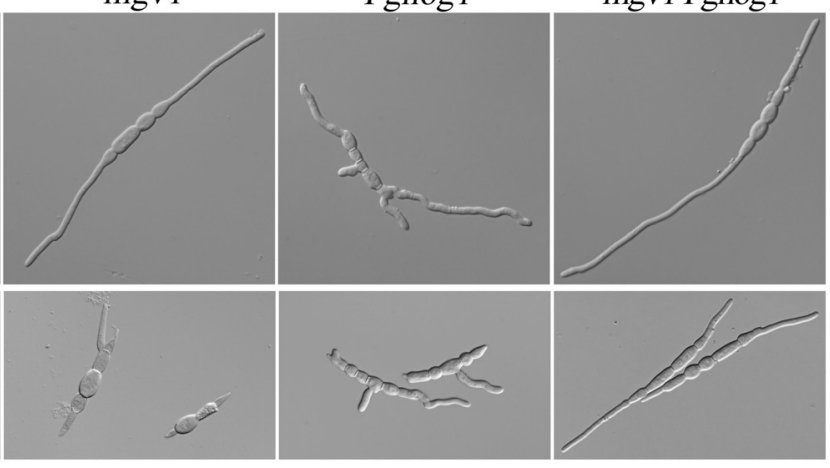

FIGURE 2 | Sensitivities of the mgv1 Fghog1 mutant to cell wall lytic enzymes and Congo red (CR). (A) Germlings harvested from $12 \mathrm{~h}$ yeast extract-peptone-dextrose medium (YEPD) cultures of the marked strains were examined after digestion with a mixture of lytic enzymes for 30 min. In comparison with the mgv1 mutant, the mgv1 Fghog1 double mutant released fewer protoplasts. Bar = 50 $\mu \mathrm{m}$. (B) Five-day-old cultures of the marked strains grown on $\mathrm{CM}$ with $300 \mu \mathrm{g} / \mathrm{ml}$ of CR. The mgv1 Fghog1 mutant grew faster than the mgv1 mutant. (C) Conidia were examined for germination and germ tube growth after incubation in liquid YEPD with or without $15 \mu \mathrm{g} / \mathrm{ml}$ of $\mathrm{CR}$ for $6 \mathrm{~h}$. Bar $=20 \mu \mathrm{m}$.

TABLE 2 | Different responses of the mutants under various stresses.

\begin{tabular}{|c|c|c|c|c|c|}
\hline \multirow[t]{2}{*}{ Strains } & \multirow{2}{*}{$\begin{array}{c}\text { Growth inhibition } \\
\text { rate }(\%)^{\mathrm{a}} \\
300 \mu \mathrm{g} / \mathrm{mL} \text { CR }\end{array}$} & \multicolumn{4}{|c|}{ Germination rate $(\%)$ b } \\
\hline & & $\begin{array}{c}\text { CR } \\
(15 \mu \mathrm{g} / \mathrm{mL})\end{array}$ & $\begin{array}{c}\text { CFW } \\
(7.5 \mu \mathrm{g} / \mathrm{mL})\end{array}$ & $\begin{array}{l}\text { Fludioxonil } \\
\text { (5 } \mu \mathrm{g} / \mathrm{mL})\end{array}$ & $\begin{array}{c}\mathrm{NaCl} \\
(0.7 \mathrm{M})\end{array}$ \\
\hline $\mathrm{PH}-1$ (WT) & $23.9 \pm 3.7^{\mathrm{C} *}$ & $99.7 \pm 0.5^{A}$ & $99.2 \pm 0.7^{A}$ & $38.8 \pm 1.8^{\mathrm{B}}$ & $98.9 \pm 0.9^{A}$ \\
\hline C6 (mgv1) & $74.0 \pm 1.4^{\mathrm{A}}$ & $14.2 \pm 4.8^{C}$ & $0.9 \pm 0.7^{C}$ & $0^{C}$ & $97.0 \pm 0.2^{\mathrm{A}}$ \\
\hline HG15 (Fghog1) & $43.7 \pm 1.8^{\mathrm{B}}$ & $99.1 \pm 1.5^{\mathrm{A}}$ & $99.0 \pm 1.7^{\mathrm{A}}$ & $99.2 \pm 0.8^{A}$ & $94.6 \pm 1.8^{\mathrm{A}}$ \\
\hline MH18 (mgv1 Fghog1) & $41.9 \pm 2.9^{\mathrm{B}}$ & $78.8 \pm 3.7^{\mathrm{B}}$ & $40.4 \pm 4.0^{\mathrm{B}}$ & $98.5 \pm 0.1^{\mathrm{A}}$ & $81.6 \pm 0.3^{B}$ \\
\hline
\end{tabular}

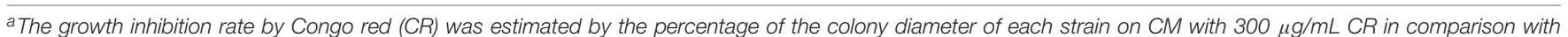

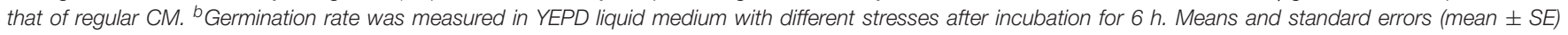

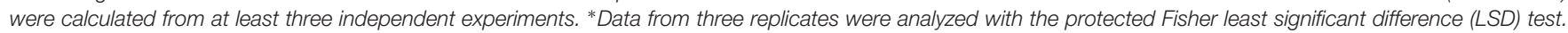
Different letters indicate statistically significant difference $(P=0.05)$.

\section{The Sensitivity of the mgv1 Mutant to Elevated Temperatures Also Is Suppressed by Deletion of FgHOG1}

Another phenotype of the $m g v 1$ mutant related to its CWI defect is increased sensitivity to elevated temperatures (Hou et al., 2002).
When germlings from $18 \mathrm{~h}$ YEPD cultures grown at $25^{\circ} \mathrm{C}$ were shifted to $32^{\circ} \mathrm{C}$ and incubated for another $6 \mathrm{~h}$, swollen hyphal tips and empty hyphal compartments were often observed in the mgv1 mutant (Figure 4). However, we failed to observe swollen hyphal tips and empty hyphal compartments in germlings of 


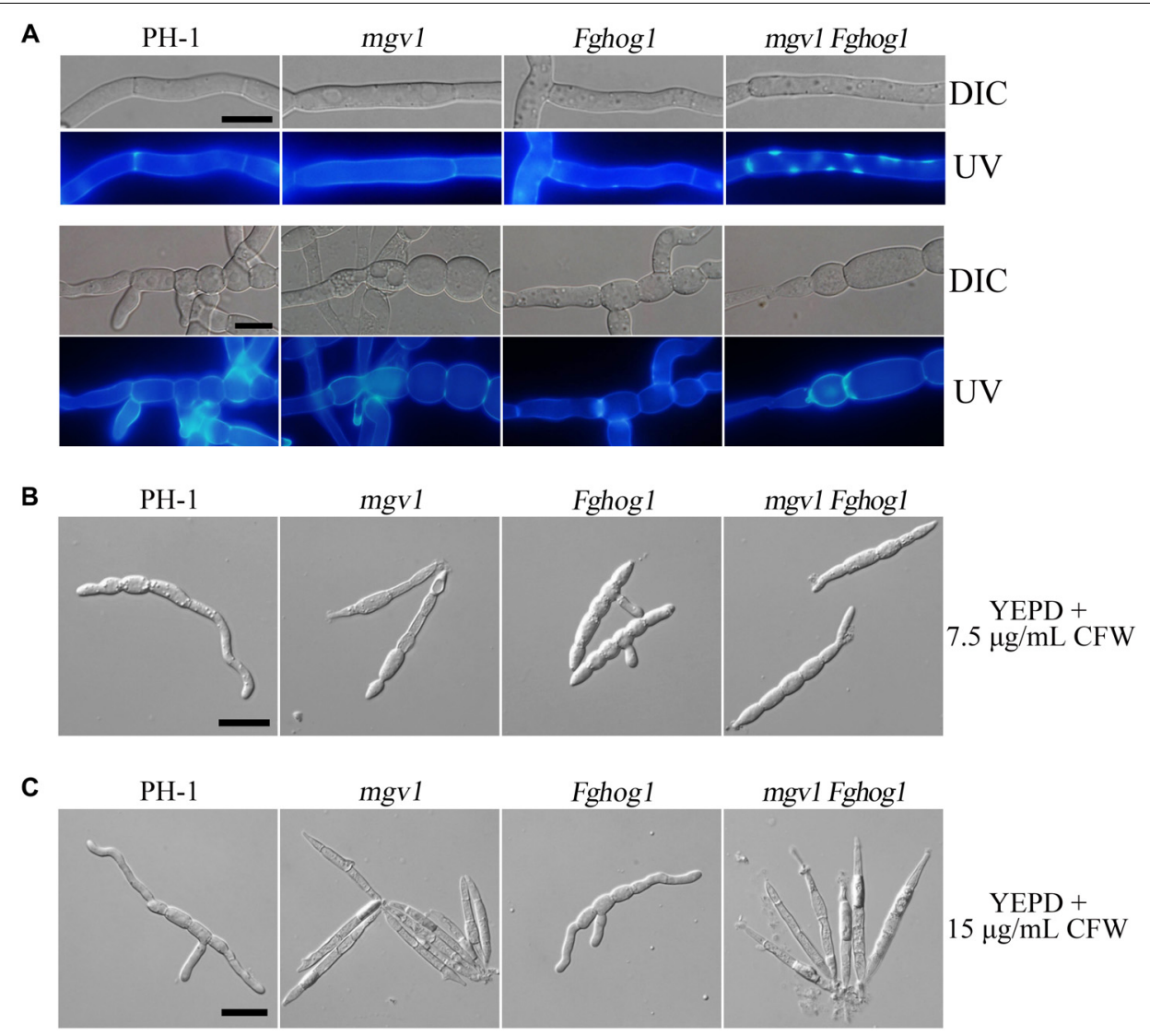

FIGURE 3 | Cell wall deposition is not normal in the mgv1 Fghog1 mutant. (A) Germlings of the wild type, mgv1, Fghog1, and mgv1 Fghog1 strains from $12 \mathrm{~h}$ YEPD cultures were stained with $50 \mu \mathrm{g} / \mathrm{ml}$ Calcofluor white (CFW) and examined by different interference contrast (DIC) and epifluorescence microscopy. Bright-staining spots were observed in the germ tubes (upper panel) but not in conidium compartments (lower panel) of the Fghog1 and double mutants. Bar = $10 \mu \mathrm{m}$. (B) Conidia of the same set of strains were incubated in liquid YEPD with $7.5 \mu \mathrm{g} / \mathrm{ml}$ of CFW for $6 \mathrm{~h}$ and were examined by DIC and epifluorescence microscopy. Extreme swelling and empty conidia compartments were observed in the mgv1 mutant. Bar $=20 \mu \mathrm{m}$. (C) Conidia of the same set of strains were incubated in liquid YEPD with $7.5 \mu \mathrm{g} / \mathrm{ml}$ of CFW for $6 \mathrm{~h}$ and were examined by DIC and epifluorescence microscopy. Most conidia of both mgv1 and mgv1 Fghog1 mutants showed empty conidia compartments. Bar $=20 \mu \mathrm{m}$.

the mgv1 Fghog1 mutant (Figure 4), suggesting that deletion of $F g H O G 1$ also could partially suppress the sensitivity of $m g v 1$ to elevated temperatures.

\section{The mgv1 Fghog1 Mutant Is More Tolerant to Fludioxonil Than the mgv1 Mutant}

Like in other filamentous fungi, the FgHogl pathway is involved in resistance to fludioxonil fungicides (Segorbe et al., 2017). In the presence of $0.1 \mu \mathrm{g} / \mathrm{ml}$ fludioxonil, colony growth was significantly inhibited in the wild type and totally blocked in the mgv1 mutant, but not affected in the Fghog1 mutant (Figure $\mathbf{5 A}$ ). Under the same conditions, the mgv1 Fghogl double mutant could grow and form colonies that were smaller than those of the Fghog1 mutant (Figure 5A). These results indicate that deletion of FgHOG1 could confer tolerance to fludioxonil in the mgv1 mutant. Interestingly, unlike the Fghog1 mutant, the double mutant grew faster on regular $\mathrm{CM}$ than on $\mathrm{CM}$ with fludioxonil, suggesting that the mgv1 Fghog1 mutant is still sensitive to fludioxonil. Therefore, Mgv1 also plays a role in tolerance against fludioxonil in F. graminearum, possibly by strengthening the cell wall.

We then assayed the effects of fludioxonil treatment on conidial germination and germ tube growth. After incubation for $6 \mathrm{~h}$ in YEPD with $5 \mu \mathrm{g} / \mathrm{ml}$ fludioxonil, conidia swelled but germinated with a low rate $(38.8 \%)$ in the wild type (Table 2). In the mgvl mutant, no germination was observed, but some of its conidium compartments had ballooned (large swelling) or became empty (Figure 5B and Table 2). The presence of fludioxonil had no obvious effects on germination in the Fghog1 and mgv1 Fghog1 mutants (Figure 5B and Table 2). After incubation for $12 \mathrm{~h}$, majority of conidia produced short germ tubes, and some conidium compartments became empty in the wild type. In the mgv1 mutant, more conidium compartments had ballooned or became empty, but most conidia failed to germinate (Figure 5B). These results indicate that the mgv1 mutant had increased sensitivity to fludioxonil compared to the wild type, and the increase was 


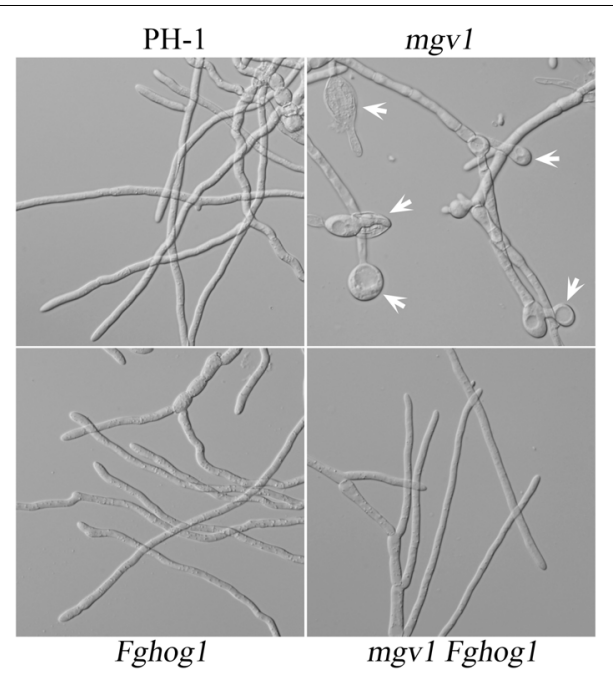

FIGURE 4 | Sensitivity of mgr 1 and mgv1 Fghog1 mutants to elevated temperature. Germlings of the marked strains from $18 \mathrm{~h}$ YEPD cultures were shifted to $32^{\circ} \mathrm{C}$ and incubated for another $6 \mathrm{~h}$. Swollen hyphal tips (marked with arrows) were abundant in the mgv1 mutant but not in other strains. However, the germ tubes of Fghog1 tended to curve and had irregular swelling that was different from the swollen hypha tips of $m g v 1$. Bar $=20 \mu \mathrm{m}$.

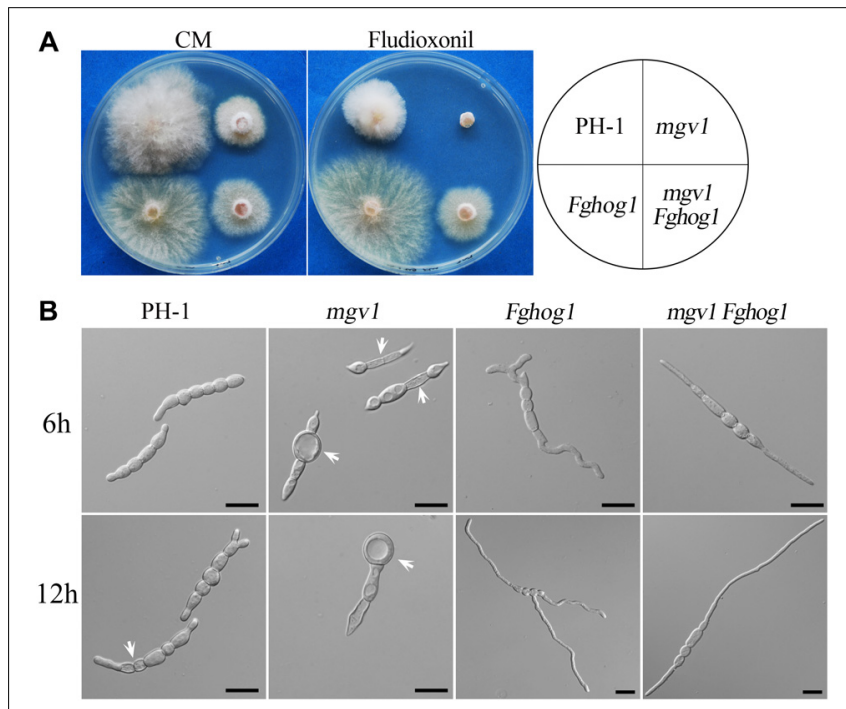

FIGURE 5 | Suppression of the hypersensitivity of $m g v 1$ to fludioxonil by deletion of FgHOG1. (A) Three-day-old cultures of PH-1, mgv1, Fghog1, and mgv1 Fghog1 strains grown on $\mathrm{CM}$ with $0.1 \mu \mathrm{g} / \mathrm{ml}$ fludioxonil at $25^{\circ} \mathrm{C}$. The double mutant was able to grow but formed smaller colonies than the Fghog1 mutant. (B) Conidia of the same set of strains were incubated in YEPD with $5 \mu \mathrm{g} / \mathrm{ml}$ fludioxonil for 6 and $12 \mathrm{~h}$ before examination. Bar $=20 \mu \mathrm{m}$. Treatment with such a low concentration of fludioxonil caused the swelling and burst of conidium compartments in the mgv1 mutant but not in other strains.

suppressed by deletion of FgHOG1. Because fludioxonil is fungicidal by overstimulating the HOG pathway and resulting in a rise in intracellular turgor (Zhang et al., 2002), the increased sensitivity of the mgv1 mutant may be directly related to its cell wall defects.
The mgv1 Fghog1 mutant is more tolerant to hyperosmotic stress than the Fghog1 mutant in vegetative growth but not in conidial germination: Because FgHog1 MAPK is involved in regulating the response to hyperosmotic stress, we then assayed the effects of $0.2 \mathrm{M} \mathrm{NaCl}$ on the growth of the $m g v 1$ and mgv1 Fghog1 mutants. As shown in Figure 6A, both the Fghog1 and mgv1 Fghog1 mutants were significantly inhibited in vegetative growth on a $\mathrm{CM}$ plate with $0.2 \mathrm{M} \mathrm{NaCl}$. Interestingly, the mgv1 Fghog1 double mutant formed a bigger colony than the Fghog1 mutant, which was contrary to the situation on a regular CM plate (Figure 6A), indicating that the deletion of MGV1 partially alleviated the hypersensitivity of the Fghog1 mutant against hyperosmotic stress. We also noticed that the mgv1 mutant grew faster on a CM plate supplemented with $0.2 \mathrm{M} \mathrm{NaCl}$ than on a regular CM plate (Supplementary Figure S2). When inoculated in liquid YEPD with $0.7 \mathrm{M} \mathrm{NaCl}$
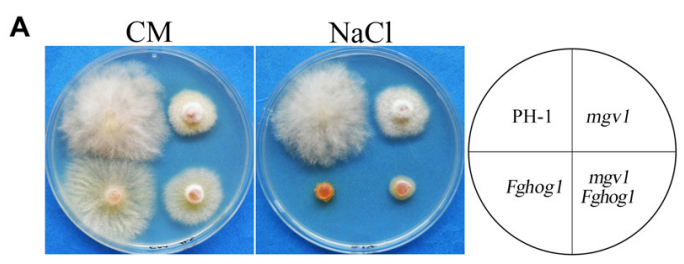

B

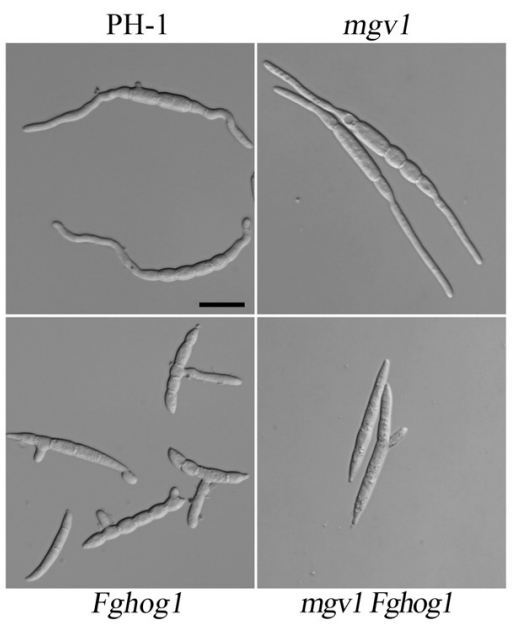

C

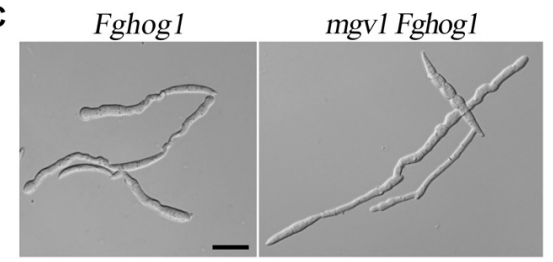

FIGURE 6 | Deletion of MGV1 caused different effects on vegetative growth and conidial germination of the Fghog1 mutant under hyperosmotic stress. (A) Three-day-old cultures of PH-1, mgv1, Fghog1, and mgv1 Fghog1 strains grown on $\mathrm{CM}$ plates with or without $0.2 \mathrm{M} \mathrm{NaCl}$. (B) Conidia of the same sets of strains were examined after incubation for $6 \mathrm{~h}$ in YEPD with $0.7 \mathrm{M} \mathrm{NaCl}$. Bar $=20 \mu \mathrm{m}$. (C) Conidia of the Fghog1 mutant and mgv1 Fghog1 mutants incubated for $12 \mathrm{~h}$ in YEPD with $0.7 \mathrm{M} \mathrm{NaCl}$ were examined by DIC. Bar $=20 \mu \mathrm{m}$. 
for $6 \mathrm{~h}$, both the $m g v 1$ and the Fghog1 mutant germinated as efficiently as the wild type $\mathrm{PH}-1$ (Table 2). In comparison with the wild type, the Fghog1 mutant but not the mgv1 mutant had shorter germ tubes and tended to have slightly swollen tips (Figure 6B). Intriguingly, under same conditions, the double mutant had a lower conidial germination rate (81.6\%) than mgv1 (97.0\%) and Fghog1 (94.6\%) (Figure 6B and Table 2). However, after incubation for $12 \mathrm{~h}$, majority of mgv1 Fghog1 germ tubes were longer than those of the Fghog1 mutant (Figure 6C). These data indicate that the deletion of MGV1 reduced the conidial germination rate of the Fghog1 mutant under hyperosmotic stress but alleviated its hyperosmotic sensitivity in vegetative growth.

\section{Deletion of FgHOG1 Increases the Phosphorylation of Gpmk1 in the mgv1 Mutant}

The phosphorylation of Gpmk1 and Mgv1 at the TEY motif could be detected with a commercially available anti-TpEY phosphorylation-specific antibody (Zhang et al., 2017). On
Western blots of total proteins isolated from vegetative hyphae harvested from $18 \mathrm{~h}$ regular YEPD cultures, the Gpmk1 and phosphorylated Gpmk1 bands were detected in all four strains, while the Mgv1 and phosphorylated Mgv1 bands were only detectable in the wild type and the Fghog1 mutant (Figure 7A). In comparison with the wild type, the phosphorylation of Gpmk1 was increased about 1.8- and 2.9-fold in the Fghog1 and mgv1 Fghog1 mutants, respectively (Figure 7A). The phosphorylation level of Gpmk1 also was higher in the double mutant than in the Fghog1 mutant. We also assayed the effects of CR treatment on MAPK phosphorylation. Hyphae harvested from $18 \mathrm{~h}$ YEPD cultures were further treated with $10 \mu \mathrm{g} / \mathrm{ml} \mathrm{CR}$ for $10 \mathrm{~min}$. On Western blots detected with the anti-TpEY antibody, phosphorylation of Gpmk1 was increased approximately 2.4- and 4.0-fold in Fghog1 and $m g v 1$ Fghog1 mutants, respectively, in comparison with the wild type (Figure 7B). However, under normal and CR stress conditions, the wild type and the Fghog1 mutant displayed similar phosphorylation levels of Mgv1 (Figures 7A,B). These results indicate that deletion of both FgHOG1 and MGV1 resulted in an increase in the activation of the Gpmk1
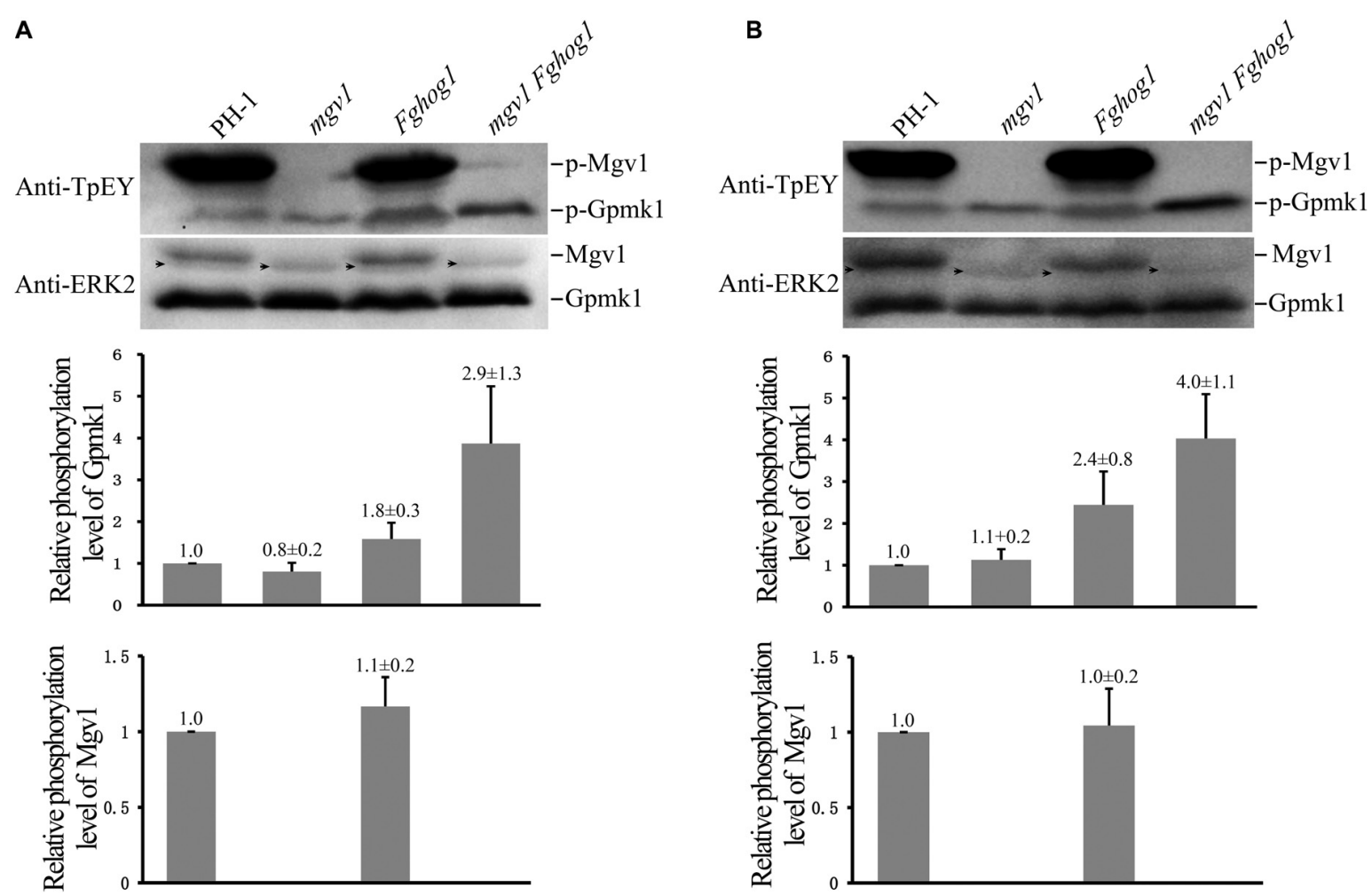

FIGURE 7 | Deletion of FgHOG1 increases the phosphorylation of Gpmk1 in the mgv1 mutant. (A) Western blots of total proteins isolated from vegetative hyphae of the marked strains harvested from $18 \mathrm{~h}$ regular YEPD cultures were detected with the anti-TpEY phosphorylation-specific antibody or anti-ERK2 MAPK antibody (upper panel). As expected, the Western bands of Mgv1 and phosphorylated Mgv1 (p-Mgv1) were not detected from the mgv1 and mgv1 Fghog1 mutants. The relative phosphorylation level of Gpmk1 ( $y$-axis) was estimated as the ratio of the phosphorylated Gpmk1 band detected with an anti-TpEY antibody to the total Gpmk1 band detected with the anti-ERK2 antibody for individual mutant strains, and compared to that of the wild type (middle panel). The relative phosphorylation level of Mgv1 ( $y$-axis) was estimated as the ratio of the phosphorylated Mgv1 band detected with an anti-TpEY antibody to the total Mgv1 band detected with the anti-ERK2 antibody, and compared to that of the wild type (lower panel). Black arrows denote non-specific bands. Error bars represent standard deviations from three independent experiments. (B) $18 \mathrm{~h}$ vegetative hyphae of the same set of strains were treated with $10 \mu \mathrm{g} / \mathrm{ml} \mathrm{CR}$ for $10 \mathrm{~min}$ and used for Western blot analyses with anti-TpEY and anti-ERK2 antibodies. Black arrows denote non-specific bands. Error bars represent standard deviations from three independent experiments. 
MAPK under normal or cell wall stress conditions, which may contribute to the suppressive effects of FgHOG1 deletion on the mgv1 mutant.

\section{Deletion of MGV1 Increased the Phosphorylation of FgHog1 in Response to Fludioxonil}

We also assayed the phosphorylation of FgHog1 with the anti-TpGY-specific antibody because FgHog1 is the only MAPK with the TGY dual-phosphorylation site in F. graminearum. On Western blots with total proteins isolated from $18 \mathrm{~h}$ regular YEPD cultures, as expected, the phosphorylated FgHog1 band was not detected with the anti-TpGY antibody in the Fghog1 and mgv1 Fghog1 mutants (Figure 8A). The phosphorylation level of FgHog1 was increased about 1.5-fold in the $m g v 1$ mutant (Figure 8A). We also conducted Western blot analysis with proteins isolated from $18 \mathrm{~h}$ hyphae that were treated with $2 \mu \mathrm{g} / \mathrm{ml}$ fludioxonil for $10 \mathrm{~min}$. In comparison with the wild type, fludioxonil treatment increased the phosphorylation of FgHog1 over 2-fold in the mgv1 mutant (Figure $\mathbf{8 B}$ ). These results indicate that deletion of $M G V 1$ had a positive effect on FgHog1 phosphorylation under both normal and fludioxonil conditions. The overstimulation of FgHog1 observed in the mgv1 mutant may lead to elevated turgor pressure.

\section{DISCUSSION}

In eukaryotic organisms, MAPK signaling pathways play important roles in sensing and responding to various external signals and overcoming host immunity defenses (Tong and Feng, 2019). The budding yeast has five MAPK pathways that regulate mating, invasive growth, CWI, HOG, and ascospore formation. Except for ascosporogenesis-specific MAPK, three MAPK cascades, consisting of MAPKs (Kss1/Hog1/Slt2), MAPK kinases (Ste7/Psb2/Mkk1), and MAPK kinase kinases (Ste11/Ssk2/Bck1), are conserved in pathogenic ascomycetes (Zhao et al., 2007). The regulatory role of each cascade comprises conserved and special aspects. In pathogenic fungi, the Kss1 MAPK is important for plant infection. This pathway is required for appressorium formation in all appressorium-forming pathogens studied, including M. oryzae, Botrytis cinerea, and Verticillium dahliae, and also plays important roles in plant penetration and invasive growth in various non-appressorium-forming pathogens (Jiang et al., 2018). The HOG pathway regulates cellular responses to high osmolarity, oxidation/heat shock, pathogenicity, and phenylpyrrole fungicides (Rispail et al., 2009). The Slt2 orthologs are well conserved in filamentous fungi, but they exhibit distinct biological functions among different species. For instance, the orthologs of Slt2 are well known to regulate the integrity of cell walls in budding yeast, M. oryzae, Ustilago maydis, and Aspergillus species (Xu et al., 1998; Carbo and Perez-Martin, 2010; Rodicio and Heinisch, 2010; Yoshimi et al., 2016) but not in B. cinerea and Colletotrichum lagenarium (Kojima et al., 2002; Rui and Hahn, 2007). Moreover, Slt2-type MAPKs are required for fungal virulence in Candida albicans and M. oryzae but not in Aspergillus fumigatus (Xu et al., 1998; Monge et al., 2006; Valiante et al., 2009). In nematode-trapping fungi, the Slt2 is also involved in mycelial trap formation (Zhen et al., 2018).

Like many other filamentous ascomycetes, F. graminearum has three well-conserved MAPK pathways that have distinct and overlapping functions in growth, development, and pathogenesis (Hou et al., 2002; Jenczmionka et al., 2003; Urban et al., 2003; Zheng et al., 2012). For vegetative growth, the CWI pathway
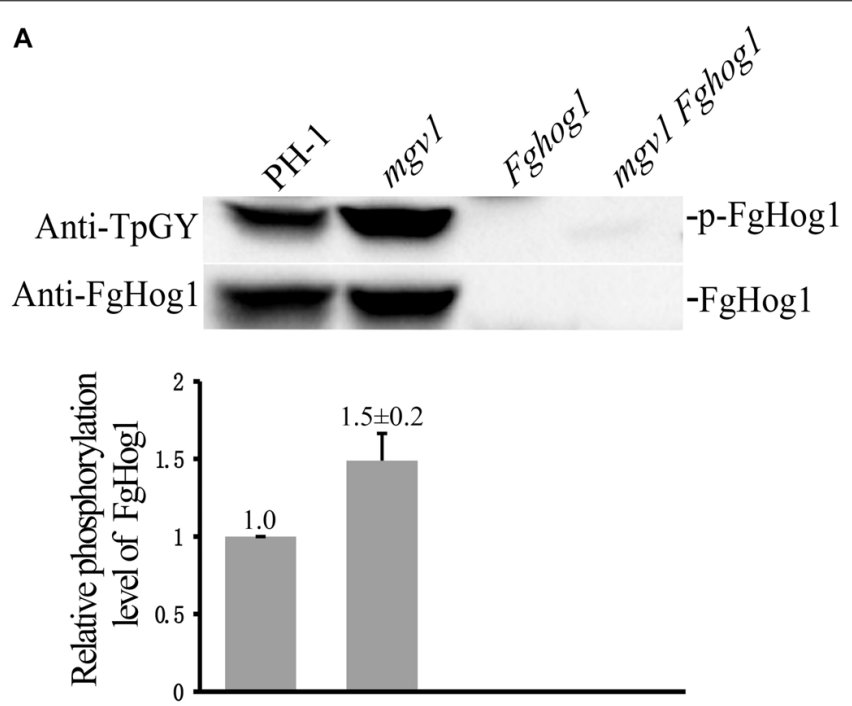

B
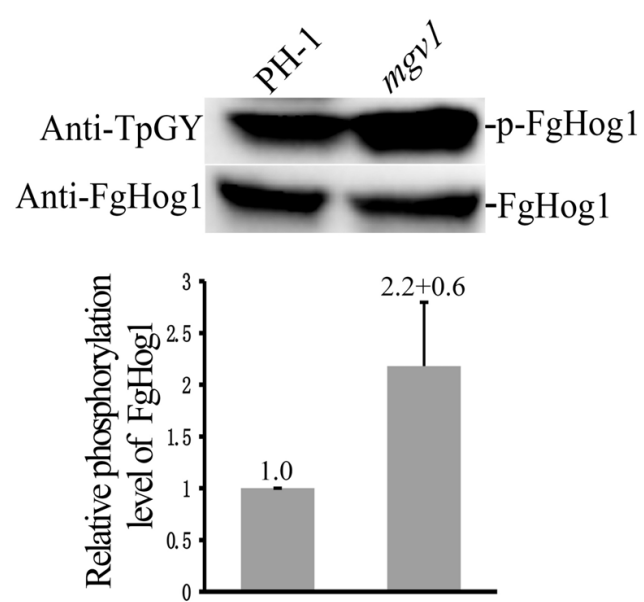

FIGURE 8 | Assays for the phosphorylation of FgHog1. (A) Western blots of total proteins isolated from vegetative hyphae harvested from 18 h regular YEPD cultures were detected with the anti-TpGY phosphorylation-specific antibody or anti-FgHog1 antibody. The FgHog1 band was not detectable in the Fghog1 and mgv1 Fghog1 mutants. In the mgv1 mutant, the phosphorylation of FgHog1 was increased by approximately 50\% in comparison with the wild type. (B) Western blots of total proteins isolated from vegetative hyphae of marked strains treated with $2 \mu \mathrm{g} / \mathrm{ml}$ fludioxonil (Flu) for 10 min. The phosphorylation of FgHog1 in the $\mathrm{mgv} 1$ mutant was two times higher than that in the wild type. 
is more important than the other MAPK pathways. Different from the Fghog1 and gpmk1 mutants, the mgv1 deletion mutant has severe growth defects (Hou et al., 2002). In filamentous ascomycetes such as B. cinerea, Neurospora crassa, and Alternaria alternata, disruption of the Slt2 ortholog also caused defects in vegetative growth (Rui and Hahn, 2007; Park et al., 2008; Yago et al., 2011). In this study, we showed that the mgv1 Fghog1 double mutant grew faster than the $m g v 1$ mutant, although it was still non-pathogenic. The suppressive effect of FgHOG1 deletion on $m g v 1$ growth indicated that these two MAPK pathways may cross talk during vegetative growth in F. graminearum. In fact, FgHOG1 may also play a role in normal hyphal growth because the Fghog1 mutant itself was slightly reduced in growth rate. The difference in the effects of FgHOG1 deletion on $m g v 1$ growth and virulence may be related to the fact that the Fghog1 mutant itself had severe defects in plant infection, although it was only slightly reduced in vegetative growth.

The mgv1 mutant is hypersensitive to cell wall stressors and lytic enzymes (Hou et al., 2002). Interestingly, the $m g v 1$ Fghog1 double mutant was less sensitive than mgv1 to cell wall lytic enzymes, CR, and CFW. In Fusarium oxysporum, the double mutant of these two MAPK genes also showed alleviated sensitivity to cell wall stressor CFW but not to CR (Segorbe et al., 2017). In F. graminearum, it is possible that some of the genes important for CWI may be downregulated in the $m g v 1$ mutant but partially rescued by deletion of FgHOG1 (directly or indirectly). In yeast, a number of genes important for cell wall synthesis and assembly are affected by deletion of SLT2 (Levin, 2011). It will be important to assay whether the $m g v 1$ mutant has similar defects in the regulation of cell wall-related genes and whether deletion of FgHOG1 impacts their expression in F. graminearum.

In comparison with the wild type, the mgv1 mutant had increased sensitivity to fludioxonil during conidial germination. Fungicide fludioxonil can increase the cytoplasmic solute concentration and intracellular turgor pressure by overstimulating the HOG pathway (Ochiai et al., 2007; Jiang et al., 2011). Deletion of FgHOG1 also improved the resistance of the mgv1 mutant to fludioxonil. Consistent with this, in F. oxysporum, the mpk1 hog1 double mutant (equivalent to the mgv1 Fghog1 mutant in F. graminearum) also has higher resistance to fludioxonil than the $m p k 1$ mutant (Segorbe et al., 2017). In F. graminearum, fludioxonil treatment likely increased the intracellular turgor pressure in the $m g v 1$ mutant, resulting in the swelling and burst of conidia and hyphae due to its cell wall defects. Deletion of FgHOG1 will avoid the fungicidal effect of fludioxonil on increasing intracellular turgor pressure. We noticed that the mgv1 Fghog1 double mutant also had increased resistance to elevated temperatures. In the budding yeast, exposure to elevated temperatures results in the accumulation of trehalose in the cytoplasm (Neves and Francois, 1992) and triggers water influx into cell, resulting in an increase in intracellular turgor pressure (Levin, 2005). Therefore, some of the suppressive effects of FgHOG1 deletion on $m g v 1$ may be simply related to avoiding an increase in intracellular turgor under certain stress conditions that is regulated by the FgHog1 MAPK pathway in F. graminearum.
This hypothesis is also supported by our result that the phosphorylation level of FgHog1 was significantly increased in the mgv1 deletion mutant, which could at least partially contribute to the defect of the mgv1 mutant. In line with this, inactivation of Slt2 in S. cerevisiae also triggers the activation of Hog 1 by downregulating a phosphatase Ptp 2 that is involved in dephosphorylation of Hog1 (Chang et al., 2013). The similar mechanism may also exist in $F$. graminearum. Intriguingly, the deletion of FgMKK1 (the upstream MAPKK of MGV1) significantly inhibits the activation of FgHog1 (Yun et al., 2014). Given that the Fgmkk1 mutant exhibited increased tolerance to fludioxonil and hypersensitivity to hyperosmotic stresses (Yun et al., 2014), which is contrary to the mgv1 mutant, we think the FgMkk1 and Mgv1 should play distinct roles in the cross talk between the HOG pathway and the CWI pathway.

While deletion of FgHOG1 was suppressive to most of the defects of the mgv1 mutant, deletion of $M G V 1$ also appeared to slightly alleviate the hyperosmotic sensitivity of the Fghog1 mutant in vegetative growth. It has been reported that in A. fumigatus, U. maydis, and Fusarium verticillioides, the BCK1, $M K K 1$, and SLT2 deletion mutants with impaired cell walls had increased tolerance to osmotic stresses (Valiante et al., 2009; Carbo and Perez-Martin, 2010; Zhang et al., 2015). In addition, we had proved that deletion of FgHOG1 only partially enhanced the cell wall and the mgv1 Fghog1 was still defective in CWI. Therefore, the alleviated sensitivity of the mgv1 Fghog1 double mutant to hyperosmotic stress could be attributed to its cell wall defect. To our surprise, the deletion of $M G V 1$ reduced the conidial germination rate of the Fghog1 mutant in YEPD with $0.7 \mathrm{M} \mathrm{NaCl}$. We noticed that the majority of conidia of the mgv1 Fghog1 mutant were unable to swell under this hyperosmotic stress condition, suggesting its failure to form enough intracellular turgor pressure. Accumulation of cytoplasmic solute could cause huge intracellular turgor pressure that is essential for conidial swelling and germination. Undoubtedly, more cytoplasmic solute could be required to generate identical intracellular turgor pressure under hyperosmotic stress conditions. It has been reported that glycerol content increased intensively at the initial swelling stage of germination, and the glycerol is synthesized from storage carbohydrates trehalose and mannitol (Morozova et al., 2002). However, it was reported that the disruption of Slt2 leads to reduced accumulation of mannitol and trehalose in Beauveria bassiana (Chen et al., 2014). Thus, we speculated that the reduced conidial germination rate of the mgv1 Fghog1 double mutant under hyperosmotic stress may be attributable to both the absence of Hog1-mediated glycerol biosynthesis and the impaired storage of trehalose and mannitol in conidia.

When assayed with an anti-TpEY phosphorylation-specific antibody, we found that the mgv1 hog1 double mutant had an increased Gpmk1 phosphorylation level in normal culture conditions or under stress conditions. Consistent with this, the mpk1 hog1 double mutant also has an over-activation of Fmk1 (ortholog of Gpmk1) in F. oxysporum (Segorbe et al., 2017). Although the CWI pathway has been considered as the main signaling pathway responsible for CWI, other signaling pathways have also been implicated in maintaining cell wall construction 
(Boorsma et al., 2004; Garcia et al., 2004; Munro et al., 2007). In the budding yeast, the Kss1 MAPK also is involved in the STE vegetative growth (SVG) pathway that promotes vegetative growth via regulating the cell wall biosynthesis (Lee and Elion, 1999; Cullen et al., 2000). In C. albicans, the Cek1 MAPK signaling pathway also plays an important role in cell wall biogenesis (Eisman et al., 2006). Gpmk1 is an ortholog of Kss1 and Cek1 in F. graminearum. It is likely that an increase in the activation of the Gpmk1 MAPK in the mgv1 Fghog1 mutant will affect the regulation of cell wall generation, which contributes to the suppressive effects of FgHOG1 deletion on the defects of the mgv1 mutant. Increased activation of Gpmk1 in the Fghog1 mutant, especially under hyperosmotic stress conditions, has been observed in an earlier study (Zheng et al., 2012). Although its exact function in CWI and hyphal growth is not clear, the gpmk1 mutant also displayed a vegetative growth defect (Wang et al., 2011). Therefore, it will be important to characterize the role of Gpmk1 in hyphal growth and cell wall biosynthesis and its relationship with $\mathrm{Mgv1}$ and FgHog1 in F. graminearum.

\section{MATERIALS AND METHODS}

\section{Fungal Strains and Culture Conditions}

The wild type strain $\mathrm{PH}-1$ and all the mutants of $F$. graminearum were routinely cultured at $25^{\circ} \mathrm{C}$ on potato dextrose agar (PDA) (200 g potato, $20 \mathrm{~g}$ glucose, and $20 \mathrm{~g}$ agar in $1 \mathrm{~L}$ water) or CM ( $10 \mathrm{~g}$ glucose, $2 \mathrm{~g}$ peptone, $1 \mathrm{~g}$ yeast extract, $1 \mathrm{~g}$ casamino acids, $6 \mathrm{~g} \mathrm{NaNO}_{3}, 0.5 \mathrm{~g} \mathrm{KCl} 0.5 \mathrm{~g} \mathrm{MgSO}_{4}$, and $1.5 \mathrm{~g} \mathrm{KH}_{2} \mathrm{PO}_{4}$ in $1 \mathrm{~L}$ water, $\mathrm{pH}$ 6.5) (Wang et al., 2018). Growth rate was measured with race tube cultures (Liu et al., 2015). Conidiation in CMC (15 g carboxymethyl cellulose, 1 g yeast extract, $0.5 \mathrm{~g} \mathrm{MgSO}_{4}, 1 \mathrm{~g}$ $\mathrm{NH}_{4} \mathrm{NO}_{3}$, and $1 \mathrm{~g} \mathrm{KH}_{2} \mathrm{PO}_{4}$ in $1 \mathrm{~L}$ water) cultures and conidial germination in YEPD $(0.3 \%$ yeast extract powder, $1 \%$ peptone, and $2 \%$ glucose) were assayed as described (Wang et al., 2012; Zheng et al., 2012). For protein extraction, $18 \mathrm{~h}$ germlings were harvested from liquid YEPD cultures.

\section{Generation of the mgv1 Fghog1 Double Mutant}

The $m g v 1$ and Fghogl single mutants were generated in previous studies (Hou et al., 2002; Zheng et al., 2012). To generate the double mutant, the split-marker approach (Catlett et al., 2003) was used to delete the FgHOG1 gene in the mgv1 mutant. The 0.91-kb upstream and 0.56-kb downstream flanking fragments of FgHOG1 gene were amplified with primer pairs $\mathrm{HOG} 1 / 1 \mathrm{~F}+2 \mathrm{R}$ and $\mathrm{HOG} 1 / 3 \mathrm{~F}+4 \mathrm{R}$, respectively. The resultant PCR products were fused with the geneticin resistance cassette amplified with primers GEN/F + GE/R and EN/F + GEN/R from the pFL2 vector (Zhou et al., 2011) and transformed into protoplasts of the mgv1 mutant as described (Wang et al., 2012). G418 (Sigma-Aldrich, St. Louis, MO, United States) was added to the final concentration of $400 \mathrm{mg} / \mathrm{mL}$ for transformant selection (Wang et al., 2018). Transformants resistant to G418 were screened by PCR with primer pairs $5 \mathrm{~F}+6 \mathrm{R}$, G850 + G852, $7 \mathrm{~F}+\mathrm{G} 856 \mathrm{R}$, and G855F $+8 \mathrm{R}$ to confirm the deletion of FgHOG1.

\section{Assays for Defects in Responses to Different Stresses}

The final concentration of $300 \mu \mathrm{g} / \mathrm{ml} \mathrm{CR}, 0.2 \mathrm{M} \mathrm{NaCl}$, or $0.1 \mu \mathrm{g} / \mathrm{ml}$ fludioxonil was added to the $\mathrm{CM}$ to assay for colony growth at $25^{\circ} \mathrm{C}$ as described (Zhang et al., 2016), and morphology in each plate was examined and photographed after incubation for the time indicated in the figure legends. To assay conidial germination, the final concentration of $15 \mu \mathrm{g} / \mathrm{ml}$ CR, $7.5 \mu \mathrm{g} / \mathrm{ml}$ CFW, $0.7 \mathrm{M}$ $\mathrm{NaCl}$, or $5 \mu \mathrm{g} / \mathrm{ml}$ fludioxonil was added to freshly harvested conidia resuspended in YEPD $\left(10^{6}\right.$ spores $\left./ \mathrm{ml}\right)$ and incubated for 6 or $12 \mathrm{~h}$ at $25^{\circ} \mathrm{C}$. In order to check the chitin distribution, germlings harvested from $12 \mathrm{~h}$ YEPD cultures were stained with $50 \mu \mathrm{g} / \mathrm{ml} \mathrm{CFW}$ for $5 \mathrm{~min}$. Germlings harvested from $18 \mathrm{~h}$ YEPD cultures grown at $25^{\circ} \mathrm{C}$ were shifted to $32^{\circ} \mathrm{C}$ and incubated for another $6 \mathrm{~h}$ to assay the effect of elevated temperature. For CWI assays, germlings harvested from $12 \mathrm{~h}$ YEPD cultures were digested with the cell wall lytic solution ( $0.1 \mathrm{~g}$ lysing enzymes, $0.5 \mathrm{~g}$ Driselase, and $10 \mathrm{mg}$ Lyticase in $20 \mathrm{ml}$ of $1.2 \mathrm{M} \mathrm{KCl}$ ) at $30^{\circ} \mathrm{C}$ for $30 \mathrm{~min}$ before examination for protoplasts (Hou et al., 2002; Yun et al., 2014). Observations were conducted under an Olympus BX-51 microscope (Olympus, Tokyo, Japan), and photographs were taken in different interference contrast (DIC) or epifluorescence modes. Each experiment was repeated at least three times independently.

\section{Plant Infection and DON Production Assays}

For plant infection assays, conidia harvested from 5-day-old CMC cultures by filtration through Miracloth were resuspended to a concentration of $1.0 \times 10^{5}$ spores $/ \mathrm{ml}$ in sterile distilled water. Flowering wheat heads of cultivar Xiaoyan 22 were drop-inoculated with $10 \mu \mathrm{l}$ of conidia suspensions at the fifth spikelet from the base of the spike as described (Gale et al., 2002). There were at least 10 replicates for each strain. Inoculated wheat heads were covered by a plastic bag for 2 days to keep humidity. Wheat spikelets with typical Fusarium head blight symptoms were examined and recorded 14 days post-inoculation (dpi) to estimate the disease index. For each strain, a DON production assay in TBI cultures was performed as described (Gardiner et al., 2009), with a competitive ELISA-based DON plate kit (Beacon Analytical Systems, Saco, ME, United States). This experiment was repeated three times.

\section{Western Blot Analysis}

Total proteins were isolated from hyphae harvested from $18 \mathrm{~h}$ YEPD cultures as described (Zhang et al., 2018). For assays with different stresses, hyphae harvested from $18 \mathrm{~h}$ YEPD were further incubated with $10 \mu \mathrm{g} / \mathrm{ml} \mathrm{CR}$ or $2 \mu \mathrm{g} / \mathrm{ml}$ fludioxonil for another $10 \mathrm{~min}$ before protein extraction. Proteins were separated on a $10 \%$ SDS-PAGE gel and transferred to nitrocellulose membranes as described (Zhang et al., 2017). The PhophoPlus p44/42 MAPK antibody kit (Cell Signaling Technology, United States) was used to detect the phosphorylation of Gpmk1 and Mgv1 as described 
(Zhang et al., 2017). Phosphorylation of FgHog1 was assayed with the Phopho p38 MAPK antibody kit (Cell Signaling Technology, United States). The expression level of Gpmk1, Mgv1, and FgHog1 was detected with the anti-Erk2 and antiFgHog1 polyclonal antibodies. Band densities were analyzed with the Image Lab ${ }^{\mathrm{TM}}$ software. Quantitative changes in the phosphorylation levels of Gpmk1, Mgv1, and FgHog1 were analyzed with the Image $\mathrm{Lab}^{\mathrm{TM}}$ software. Each experiment was repeated four times, independently.

\section{AUTHOR CONTRIBUTIONS}

GW, CJ, and J-RX conceived and designed the experiments. JR, CL, and CG performed the experiments. GW and CJ analyzed the data. GW wrote the manuscript. J-RX and CJ improved the manuscript.

\section{FUNDING}

This project was supported by grants to J-RX from NSF and USWBSI, National Natural Science Foundation of China (No. 31801684) and National Undergraduate Training Program for Innovation and Entrepreneurship (No. 201810712103).

\section{REFERENCES}

Bai, G., and Shaner, G. (2004). Management and resistance in wheat and barley to fusarium head blight. Annu. Rev. Phytopathol. 42, 135-161. doi: 10.1146/ annurev.phyto.42.040803.140340

Boenisch, M. J., Broz, K. L., Purvine, S. O., Chrisler, W. B., Nicora, C. D., Connolly, L. R., et al. (2017). Structural reorganization of the fungal endoplasmic reticulum upon induction of mycotoxin biosynthesis. Sci. Rep. 7:44296. doi: $10.1038 /$ srep 44296

Boorsma, A., de Nobel, H., ter Riet, B., Bargmann, B., Brul, S., Hellingwerf, K. J., et al. (2004). Characterization of the transcriptional response to cell wall stress in Saccharomyces cerevisiae. Yeast 21, 413-427. doi: 10.1002/yea.1109

Carbo, N., and Perez-Martin, J. (2010). Activation of the cell wall integrity pathway promotes escape from G2 in the fungus Ustilago maydis. PLoS Genet. 6:e1001009. doi: 10.1371/journal.pgen.1001009

Catlett, N. L., Lee, B. N., Yoder, O. C., and Turgeon, B. G. (2003). Split-marker recombination for efficient targeted deletion of fungal genes. Fungal. Genet. Newsl. 50, 9-11.

Chang, M., Kang, H. J., Baek, I. J., Kang, C. M., Park, Y. S., and Yun, C. W. (2013). Rck1 up-regulates Hog1 activity by down-regulating Slt2 activity in Saccharomyces cerevisiae. Biochem. Biophys. Res. Commun. 440, 119-124. doi: 10.1016/j.bbrc.2013.09.045

Chen, Y., Zhu, J., Ying, S. H., and Feng, M. G. (2014). Three mitogen-activated protein kinases required for cell wall integrity contribute greatly to biocontrol potential of a fungal entomopathogen. PLoS One 9:e87948. doi: 10.1371/journal. pone.0087948

Cullen, P. J., Schultz, J., Horecka, J., Stevenson, B. J., Jigami, Y., Sprague, G. F. Jr., et al. (2000). Defects in protein glycosylation cause SHO1-dependent activation of a STE12 signaling pathway in yeast. Genetics $155,1005-1018$.

Dixon, K. P., Xu, J. R., Smirnoff, N., and Talbot, N. J. (1999). Independent signaling pathways regulate cellular turgor during hyperosmotic stress and appressorium-mediated plant infection by Magnaporthe grisea. Plant Cell 11, 2045-2058.

Eisman, B., Alonso-Monge, R., Roman, E., Arana, D., Nombela, C., and Pla, J. (2006). The Cek1 and Hogl mitogen-activated protein kinases play complementary roles in cell wall biogenesis and chlamydospore formation in the fungal pathogen Candida albicans. Eukaryot. Cell. 5, 347-358. doi: 10.1128/ ec.5.2.347-358.2006

\section{ACKNOWLEDGMENTS}

We would like to thank Dr. Xue Zhang and Xinyu Cao for the excellent technical support and Dr. Huiquan Liu for the insightful discussions.

\section{SUPPLEMENTARY MATERIAL}

The Supplementary Material for this article can be found online at: https://www.frontiersin.org/articles/10.3389/fmicb. 2019.01073/full\#supplementary-material

FIGURE S1 | PCR assays for the confirmation of the deletion of FgHOG1 under the mgv1 mutant background. (A) The FgHOG1 locus and gene replacement construct. The FgHOG1 and NEO genes are marked with empty and black arrows, respectively. (B) A total of 18 G418-resistant mgv1 Fghog1 transformants $(\mathrm{MH} 1$ to $\mathrm{MH1} 18)$ were screened by PCR with primers $5 \mathrm{~F}$ and $6 \mathrm{R}$. (C) $\mathrm{MH} 14$, $\mathrm{MH} 17$, and $\mathrm{MH} 18$ were further verified by $P C R$ with four pairs of primers, $\mathrm{L} 1$ (5F + 6R), L2 (G850 + G852), L3 (7F + G856R), and L4 (G855F + 8R).

FIGURE S2 | The mgv1 mutant grew faster on CM containing $0.2 \mathrm{M} \mathrm{NaCl}$ than on regular CM. (A) Three-day-old cultures of mgv1 mutant grown on CM with and without $0.2 \mathrm{M} \mathrm{NaCl}$. The $m g v 1$ mutant grew faster on $\mathrm{CM}$ containing $0.2 \mathrm{M} \mathrm{NaCl}$ than on regular $\mathrm{CM}$. (B) The diagram shows the growth rate of mgv1 mutant on $\mathrm{CM}$ with and $0.2 \mathrm{M} \mathrm{NaCl}$.

Gale, L. R., Chen, L. F., Hernick, C. A., Takamura, K., and Kistler, H. C. (2002). Population analysis of Fusarium graminearum from wheat fields in eastern China. Phytopathology 92, 1315-1322. doi: 10.1094/phyto.2002.92.12.1315

Garcia, R., Bermejo, C., Grau, C., Perez, R., Rodriguez-Pena, J. M., Francois, J., et al. (2004). The global transcriptional response to transient cell wall damage in Saccharomyces cerevisiae and its regulation by the cell integrity signaling pathway. J. Biol. Chem. 279, 15183-15195. doi: 10.1074/jbc.M3129 54200

Gardiner, D. M., Kazan, K., and Manners, J. M. (2009). Nutrient profiling reveals potent inducers of trichothecene biosynthesis in Fusarium graminearum. Fungal. Genet. Biol. 46, 604-613. doi: 10.1016/j.fgb.2009.04.004

Goswami, R. S., and Kistler, H. C. (2004). Heading for disaster: Fusarium graminearum on cereal crops. Mol. Plant Pathol. 5, 515-525. doi: 10.1111/j. 1364-3703.2004.00252.x

Hou, Z. M., Xue, C. Y., Peng, Y. L., Katan, T., Kistler, H. C., and Xu, J. R. (2002). A mitogen-activated protein kinase gene (MGV1) in Fusarium graminearum is required for female fertility, heterokaryon formation, and plant infection. Mol. Plant Microbe Interact. 15, 1119-1127.

Jenczmionka, N. J., Maier, F. J., Losch, A. P., and Schafer, W. (2003). Mating, conidiation and pathogenicity of Fusarium graminearum, the main causal agent of the head-blight disease of wheat, are regulated by the MAP kinase Gpmk1. Curr. Genet. 43, 87-95. doi: 10.1007/s00294-003-0379-2

Jiang, C., Zhang, C., Wu, C., Sun, P., Hou, R., Liu, H., et al. (2016). TRI6 and TRI10 play different roles in the regulation of deoxynivalenol (DON) production by cAMP signalling in Fusarium graminearum. Environ. Microbiol. 18, 3689-3701. doi: 10.1111/1462-2920.13279

Jiang, C., Zhang, X., and Liu, H. (2018). Mitogen-activated protein kinase signaling in plant pathogenic fungi. PLoS Pathog. 14:e1006875. doi: 10.3389/fpls.2018. 0043810.1371/journal.ppat.1006875

Jiang, J., Liu, X., Yin, Y., and Ma, Z. (2011). Involvement of a velvet protein FgVeA in the regulation of asexual development, lipid and secondary metabolisms and virulence in Fusarium graminearum. PLoS One 6:e28291. doi: 10.1371/journal. pone.0028291

Kojima, K., Kikuchi, T., Takano, Y., Oshiro, E., and Okuno, T. (2002). The mitogenactivated protein kinase gene MAF1 is essential for the early differentiation phase of appressorium formation in Colletotrichum lagenarium. Mol. Plant Microbe Interact. 15, 1268-1276. doi: 10.1094/mpmi.2002.15.12.1268 
Lee, B. N., and Elion, E. A. (1999). The MAPKKK Ste11 regulates vegetative growth through a kinase cascade of shared signaling components. Proc. Natl. Acad. Sci. U.S.A. 96, 12679-12684.

Levin, D. E. (2005). Cell wall integrity signaling in Saccharomyces cerevisiae. Microbiol. Mol. Biol. Rev. 69, 262-291. doi: 10.1128/mmbr.69.2.262-291.2005

Levin, D. E. (2011). Regulation of cell wall biogenesis in Saccharomyces cerevisiae: the cell wall integrity signaling pathway. Genetics 189, 1145-1175. doi: 10.1534/ genetics.111.128264

Liu, H., Zhang, S., Ma, J., Dai, Y., Li, C., Lyu, X., et al. (2015). Two Cdc2 kinase genes with distinct functions in vegetative and infectious hyphae in Fusarium graminearum. PLoS Pathog. 11:e1004913. doi: 10.1371/journal.ppat.100548510. 1371/journal.ppat.1004913

Merzendorfer, H. (2013). Chitin synthesis inhibitors: old molecules and new developments. Insect Sci. 20, 121-138. doi: 10.1111/j.1744-7917.2012.01535.x

Monge, R. A., Roman, E., Nombela, C., and Pla, J. (2006). The MAP kinase signal transduction network in Candida albicans. Microbiology 152(Pt 4), 905-912. doi: 10.1099/mic.0.28616-0

Morozova, E. V., Kozlov, V. P., Tereshina, V. M., Memorskaia, A. S., and Feofilova, E. P. (2002). Changes in lipid composition and carbohydrate composition of Aspergillus niger conidia during germination. Prikl. Biokhim. Mikrobiol. 38, 149-154.

Munro, C. A., Selvaggini, S., de Bruijn, I., Walker, L., Lenardon, M. D., Gerssen, B., et al. (2007). The PKC, HOG and $\mathrm{Ca}^{2+}$ signalling pathways co-ordinately regulate chitin synthesis in Candida albicans. Mol. Microbiol. 63, 1399-1413. doi: $10.1111 / j .1365-2958.2007 .05588 . x$

Neves, M. J., and Francois, J. (1992). On the mechanism by which a heat shock induces trehalose accumulation in Saccharomyces cerevisiae. Biochem. J. $288(\mathrm{Pt}$ 3), 859-864.

Ochiai, N., Tokai, T., Nishiuchi, T., Takahashi-Ando, N., Fujimura, M., and Kimura, M. (2007). Involvement of the osmosensor histidine kinase and osmotic stress-activated protein kinases in the regulation of secondary metabolism in Fusarium graminearum. Biochem. Biophys. Res. Commun. 363, 639-644. doi: 10.1016/j.bbrc.2007.09.027

Park, G., Pan, S., and Borkovich, K. A. (2008). Mitogen-activated protein kinase cascade required for regulation of development and secondary metabolism in Neurospora crassa. Eukaryot. Cell 7, 2113-2122. doi: 10.1128/ec. 00466-07

Rispail, N., Soanes, D. M., Ant, C., Czajkowski, R., Grunler, A., Huguet, R., et al. (2009). Comparative genomics of MAP kinase and calcium-calcineurin signalling components in plant and human pathogenic fungi. Fungal. Genet. Biol. 46, 287-298. doi: 10.1016/j.fgb.2009.01.002

Rodicio, R., and Heinisch, J. J. (2010). Together we are strong - cell wall integrity sensors in yeasts. Yeast 27, 531-540. doi: 10.1002/yea.1785

Rui, O., and Hahn, M. (2007). The Slt2-type MAP kinase Bmp3 of Botrytis cinerea is required for normal saprotrophic growth, conidiation, plant surface sensing and host tissue colonization. Mol. Plant Pathol. 8, 173-184. doi: 10.1111/j.13643703.2007.00383.x

Saito, H., and Tatebayashi, K. (2004). Regulation of the osmoregulatory HOG MAPK cascade in yeast. J. Biochem. 136, 267-272. doi: 10.1093/jb/mvh135

Segorbe, D., Di Pietro, A., Perez-Nadales, E., and Turra, D. (2017). Three Fusarium oxysporum mitogen-activated protein kinases (MAPKs) have distinct and complementary roles in stress adaptation and cross-kingdom pathogenicity. Mol. Plant Pathol. 18, 912-924. doi: 10.1111/mpp.12446

Tong, S. M., and Feng, M. G. (2019). Insights into regulatory roles of MAPKcascaded pathways in multiple stress responses and life cycles of insect and nematode mycopathogens. Appl. Microbiol. Biotechnol. 103, 577-587. doi: 10. 1007/s00253-018-9516-1

Trail, F. (2009). For blighted waves of grain: Fusarium graminearum in the postgenomics era. Plant Physiol. 149, 103-110. doi: 10.1104/pp.108.129684

Urban, M., Mott, E., Farley, T., and Hammond-Kosack, K. (2003). The Fusarium graminearum MAP1 gene is essential for pathogenicity and development of perithecia. Mol. Plant Pathol. 4, 347-359. doi: 10.1046/j.13643703.2003.00183.x

Valiante, V., Jain, R., Heinekamp, T., and Brakhage, A. A. (2009). The MpkA MAP kinase module regulates cell wall integrity signaling and pyomelanin formation in Aspergillus fumigatus. Fungal. Genet. Biol. 46, 909-918. doi: 10.1016/j.fgb. 2009.08.005

Wang, C., Zhang, S., Hou, R., Zhao, Z., Zheng, Q., Xu, Q., et al. (2011). Functional analysis of the kinome of the wheat scab fungus Fusarium graminearum. PLoS Pathog. 7:e1002460. doi: 10.1371/journal.ppat.1002460
Wang, G., Sun, P., Gong, Z., Gu, L., Lou, Y., Fang, W., et al. (2018). Srk1 kinase, a SR protein-specific kinase, is important for sexual reproduction, plant infection and pre-mRNA processing in Fusarium graminearum. Environ. Microbiol. 20, 3261-3277. doi: 10.1007/978-1-4939-8724-5_810.1111/1462-2920.14299

Wang, G. H., Wang, C. F., Hou, R., Zhou, X. Y., Li, G. T., Zhang, S. J., et al. (2012). The AMT1 arginine methyltransferase gene is important for plant infection and normal hyphal growth in Fusarium graminearum. PLoS One 7:e38324. doi: 10.1371/journal.pone.0038324

Xu, J. R., and Hamer, J. E. (1996). MAP kinase and cAMP signaling regulate infection structure formation and pathogenic growth in the rice blast fungus Magnaporthe grisea. Genes Dev. 10, 2696-2706.

Xu, J. R., Staiger, C. J., and Hamer, J. E. (1998). Inactivation of the mitogenactivated protein kinase Mps1 from the rice blast fungus prevents penetration of host cells but allows activation of plant defense responses. Proc. Natl. Acad. Sci. U.S.A. 95, 12713-12718.

Yago, J. I., Lin, C. H., and Chung, K. R. (2011). The SLT2 mitogen-activated protein kinase-mediated signalling pathway governs conidiation, morphogenesis, fungal virulence and production of toxin and melanin in the tangerine pathotype of Alternaria alternata. Mol. Plant Pathol. 12, 653-665. doi: 10.1111/ j.1364-3703.2010.00701.x

Yoshimi, A., Miyazawa, K., and Abe, K. (2016). Cell wall structure and biogenesis in Aspergillus species. Biosci. Biotechnol. Biochem. 80, 1700-1711. doi: 10.1080/ 09168451.2016.1177446

Yun, Y., Liu, Z., Zhang, J., Shim, W. B., Chen, Y., and Ma, Z. (2014). The MAPKK FgMkk1 of Fusarium graminearum regulates vegetative differentiation, multiple stress response, and virulence via the cell wall integrity and high-osmolarity glycerol signaling pathways. Environ. Microbiol. 16, 2023-2037. doi: 10.1111/ 1462-2920.12334

Zhang, C., Wang, J., Tao, H., Dang, X., Wang, Y., Chen, M., et al. (2015). FvBck1, a component of cell wall integrity MAP kinase pathway, is required for virulence and oxidative stress response in sugarcane pokkah boeng pathogen. Front. Microbiol. 6:1096. doi: 10.3389/fmicb.2015.01096

Zhang, S., Jiang, C., Zhang, Q., Qi, L., Li, C., and Xu, J. R. (2016). Thioredoxins are involved in the activation of the PMK1 MAP kinase pathway during appressorium penetration and invasive growth in Magnaporthe oryzae. Environ. Microbiol. 18, 3768-3784. doi: 10.1111/1462-2920.13315

Zhang, X., Bian, Z., and Xu, J. R. (2018). Assays for MAP kinase activation in Magnaporthe oryzae and other plant pathogenic fungi. Methods Mol. Biol. 1848, 93-101. doi: 10.1007/978-1-4939-8724-5_8

Zhang, X., Liu, W., Li, Y., Li, G., and Xu, J. R. (2017). Expression of HopAI interferes with MAP kinase signalling in Magnaporthe oryzae. Environ. Microbiol. 19, 4190-4204. doi: 10.1111/1462-2920.13884

Zhang, Y., Lamm, R., Pillonel, C., Lam, S., and Xu, J. R. (2002). Osmoregulation and fungicide resistance: the Neurospora crassa os-2 gene encodes a HOG1 mitogen-activated protein kinase homologue. Appl. Environ. Microbiol. 68, 532-538.

Zhao, X. H., Mehrabi, R., and Xu, J. R. (2007). Mitogen-activated protein kinase pathways and fungal pathogenesis. Eukaryot. Cell 6, 1701-1714.

Zhen, Z., Xing, X., Xie, M., Yang, L., Yang, X., Zheng, Y., et al. (2018). MAP kinase Slt2 orthologs play similar roles in conidiation, trap formation, and pathogenicity in two nematode-trapping fungi. Fungal. Genet. Biol. 116, 42-50. doi: 10.1016/j.fgb.2018.04.011

Zheng, D., Zhang, S., Zhou, X., Wang, C., Xiang, P., Zheng, Q., et al. (2012). The FgHOG1 pathway regulates hyphal growth, stress responses, and plant infection in Fusarium graminearum. PLoS One 7:e49495. doi: 10.1371/journal. pone. 0049495

Zhou, X., Li, G., and Xu, J. R. (2011). Efficient approaches for generating GFP fusion and epitope-tagging constructs in filamentous fungi. Methods Mol. Biol. 722, 199-212. doi: 10.1007/978-1-61779-040-9_15

Conflict of Interest Statement: The authors declare that the research was conducted in the absence of any commercial or financial relationships that could be construed as a potential conflict of interest.

Copyright (c) 2019 Ren, Li, Gao, Xu, Jiang and Wang. This is an open-access article distributed under the terms of the Creative Commons Attribution License (CC BY). The use, distribution or reproduction in other forums is permitted, provided the original author(s) and the copyright owner(s) are credited and that the original publication in this journal is cited, in accordance with accepted academic practice. No use, distribution or reproduction is permitted which does not comply with these terms. 